Schweizerische Zeitschrift für

Ganzheitsmedizin

Swiss Journal of Integrative Medicine

Christoph Bachmann

\section{Chronisch-venöse Insuffizienz}

Die CVI ist eine weit verbreitete chronische Gefässerkrankung mit altersabhängiger Häufigkeit. Risikofaktoren wie Vererbung, Schwangerschaft, Nikotinabusus, stehende Berufe und Übergewicht sind schon lange bekannt [1]. Eine CVI entsteht durch die Erschlaffung von Venen. Als Folge können die Venenklappen nicht mehr vollständig schliessen und das Blut in Richtung Herz transportieren. Daraus entsteht ein Überdruck in den Beinvenen, der via eine pathologische Kaskade in eine Ödembildung im Knöchelbereich mündet. Die Patienten verspüren mit den Ödemen Spannungen, Juckreiz und Schmerzen in den Beinen. Ohne entsprechende Behandlung können daraus ein Ulcus cruris und/ oder Krampfadern entstehen [2].

\section{Therapie}

Als nichtmedikamentöse Therapie einer CVI kommen eine Änderung der Lebensgewohnheiten und/oder eine Kompressionstherapie mit Kompressionstrümpfen infrage.

Für eine medikamentöse Therapie werden orale oder topische Venentherapeutika - oder eine Kombination von beiden - eingesetzt. Zu den synthetischen Wirkstoffen gehören Heparine, Pentoxifyllin, Naftazon sowie Calciumdobesilat. Weiter werden isolierte Naturstoffe wie Oxerutin, Hesperidin und Troxerutin verwendet.

\section{Aus der Forschung}

Schweiz Z Ganzheitsmed 2017;29:201-203

DOI: $10.1159 / 000477787$

\section{Phytotherapie bei chronisch-venöser Insuffizienz: Wirksamkeit von rotem Weinlaub und von Rosskastanie klinisch belegt}

Die chronisch-venöse Insuffizienz (CVI) ist eine Venenerkrankung, die mit verschiedenen pflanzlichen Extrakten behandelt werden kann. Nebst Arzneipflanzen, deren Wirksamkeit nicht oder kaum dokumentiert ist, stehen zur Behandlung von Venenerkrankungen - in erster Linie der CVI - Extrakte aus der Rosskastanie und dem roten Weinlaub im Fokus. Verschiedene klinische Studien zeigen ihre Wirksamkeit.

\section{Arzneipflanzen}

Zur Behandlung oder als Begleittherapie einer CVI stehen verschiedene Arzneipflanzen zur Verfügung: Das sind:

- Mäusedorn (Ruscus aculeatus),

- Arnika (Arnica montana),

- Rosskastanie (Aesculus hippocastanum) (Abb. 1),

- Rotes Weinlaub (Vitis vinifera) (Abb. 2),

- Steinklee (Melilotus officinalis).

Die Datenlage über die Wirksamkeit ist jedoch sehr unterschiedlich. Das Herbal Medicinal Products Committee (HMPC), eine Institution der European Medicines Agency (EMA), hat Monographien für Steinklee und Mäusedorn erstellt [3]. Die beiden Monographien machen aber bei beiden Arzneidrogen nur Angaben über «traditional use», d.h. keine Angaben über «well-established use».

\section{Mäusedorn-Extrakt}

Trotzdem gibt es eine klinische Studie mit positivem Resultat für den Mäusedorn-Extrakt [4]. Diese rando-

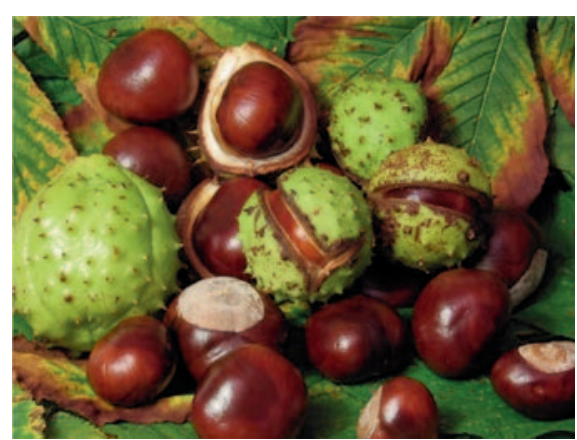

Abb. 1. Samen von Rosskastanien (Aesculus hippocastanum).

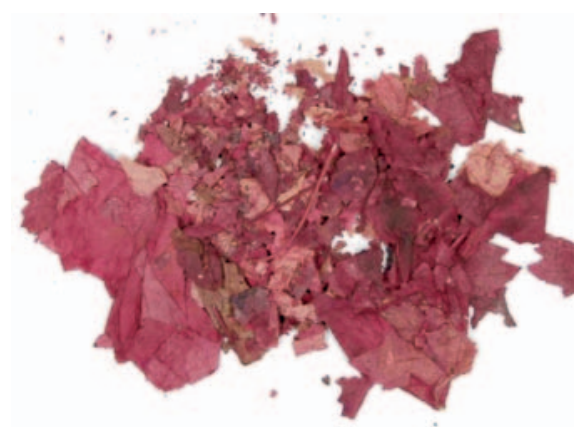

Abb. 2. Getrocknete rote Weinblätter (Vitis vinifera).

misierte, placebokontrollierte Doppelblindstudie wurde 2002 mit 166 Frauen durchgeführt, die seit Jahren an einer CVI litten. Primäre Zielvariable war das Volumen der Unter-

\section{KARGER}

() 2017 S. Karger GmbH, Freiburg

Fax +497614520714 
schenkel; sekundäre Zielvariable waren der Beinumfang an der Wade bzw. am Fussgelenk sowie das subjektive Empfinden und die Lebensqualität der Probandinnen. Bei allen erhobenen Parametern erwies sich die VerumGruppe gegenüber der Placebo-Gruppe als signifikant überlegen.

In der Schweiz gibt es je ein topisches und ein orales Kombinationspräparat mit Mäusedorn-Extrakt und je einem zweiten Wirkstoff zur Behandlung von Venenproblemen wie CVI.

\section{Arnika}

Die HMPC-Monographie von $A$. montana macht keinerlei Angaben über eine volksmedizinisch oder klinisch belegte Wirksamkeit von Arnika bei Venenproblemen. Trotzdem gibt es Hinweise auf eine Wirksamkeit bei topischer Anwendung [5]. (Diese Quelle konnte aber nicht auf ihren Gehalt überprüft werden.)

\section{Rosskastanie}

Viel mehr Evidenz über die Wirksamkeit von Rosskastanie und rotem Weinlaub bieten die verschiedenen Studien, die publiziert wurden. Mit $A$. hippocastanum wurden einige Studien durchgeführt, welche die Wirksamkeit dieser Arzneipflanze bei CVI belegen.

\section{Vergleich zur Kompression}

1996 wurde eine Studie publiziert, welche einen Vergleich zwischen der Kompressionstherapie und der oralen Anwendung von Rosskastanien-Extrakt mit Placebo machte [6]. Bei dieser randomisierten, placebokontrollierten, einseitig blinden Parallelstudie nahmen 240 Patienten mit CVI zweimal täglich Rosskastaniensamen-Trockenextrakt mit $50 \mathrm{mg}$ Aescin $(\mathrm{n}=95)$ oder Placebo $(n=46)$ ein, oder sie wurden mit einer Kompressionsthera- pie behandelt $(n=99)$. Zielparameter waren die Reduktion der Ödeme und die Verträglichkeit.

Nach 12 Behandlungswochen betrug die Ödemverminderung in der Rosskastanien-Gruppe im Mittel 43,8 $\mathrm{ml}$ und in der KompressionstherapieGruppe 46,7 ml. In der Placebo-Gruppe wurde eine Zunahme von 9,8 ml beobachtet. Die Verminderung war in den beiden Behandlungsgruppen gegenüber Placebo signifikant überlegen (Rosskastanie: $\mathrm{p}=0,005$; Kompressionstherapie: $p=0,002$ ). Beide Behandlungsarten erwiesen sich als gleichwertig $(\mathrm{p}=0,001)$. Damit erwiesen sich diese beiden Behandlungsarten als alternative, wirksame Therapien bei CVI. Eine weitere Studie bestätigte die Wirksamkeit dieser Dosierung von $50 \mathrm{mg}$ Aescin [7].

\section{Cochrane-Review}

Die Wirksamkeit von Rosskastanien-Extrakt wurde sogar in einem Review der Cochrane Collaboration untersucht - mit einem positiven Resultat [8]. Dabei untersuchten die Autoren die Wirksamkeit von Rosskastanien-Extrakt bei CVI gegenüber Placebo oder einer etablierten Behandlungsweise. Kombinationspräparate von Rosskastanien-Extrakt und weiteren Wirkstoffen wurden nicht berücksichtigt.

17 Studien, die zwischen 1976 und 2002 publiziert wurden, entsprachen den Einschlusskriterien der Autoren. 10 Studien waren placebokontrolliert, 2 Studien verglichen RosskastanienExtrakt gegenüber Placebo und gegenüber Kompressionstherapie, und 5 Studien wurden als Vergleichsstudien gegenüber $\mathrm{O}-\beta$-Hydroxyethyl-Rutosid $(\mathrm{n}=4)$ bzw. gegenüber Pycnogenol $(\mathrm{n}=1)$ angelegt.

Zielvariablen dieser 17 Studien waren Beinschmerzen, Ödeme, Pruritus, Beinvolumen und Beinumfang. Weiter wurden unerwünschte Ereignisse erfasst.

Die Resultate der 17 Studien zeigen, dass Rosskastanien-Extrakte zur Be- handlung von CVI verglichen mit Placebo, einer Standardtherapie oder einer Kompressionstherapie wirksam sind. Es wurden nur wenige und zudem unbedeutende unerwünschte Ereignisse festgestellt.

Die Cochrane-Autoren schlossen aus diesen Resultaten, dass Rosskastanien-Extrakte eine wirksame und sichere Therapiemöglichkeit zur Behandlung von CVI sind.

Diese uneingeschränkte positive Beurteilung einer phytotherapeutischen Behandlungsweise durch einen Cochrane-Review ist aussergewöhnlich! Die Cochrane Collaboration verhält sich der Phytotherapie gegenüber sehr kritisch, und viele Cochrane-Reviews über phytotherapeutische Behandlungsweisen führten trotz bestehender Evidenz zu einem negativen Resultat.

\section{Rotes Weinlaub}

Bei der phytotherapeutischen Behandlung von CVI spielt das rote Weinlaub eine ebenso wichtige Rolle wie die Rosskastanie. Beim roten Weinlaub handelt es sich um die Blätter derselben Pflanze, aus der Wein hergestellt wird.

Hauptinhaltsstoffe sind Flavonoide wie Quercetin, Isoquercitrin und Glukuronide. In-vitro-Studien zeigen, dass diese Stoffe eine antiödematöse Wirkung besitzen, indem sie die Wände der Kapillaren stärken. Dadurch wird die Permeabilität der Gefässwände vermindert, was den Übertritt von Wasser in das Interstitium hemmt.

Auch mit Weinlaub-Extrakten wurden klinische Studien durchgeführt, welche die Wirksamkeit dokumentierten.

\section{Kiesewetter et al., 2000: AS 195}

Kiesewetter et al. [9] publizierten 2000 eine Studie, die mit dem standardisierten Weinlaub-Extrakt AS 195 durchgeführt wurde. Bei dieser randomisierten kontrollierten Studie erhiel- 
ten 260 Probanden mit CVI (Stadium I-II) während 12 Wochen einmal täglich entweder $360 \mathrm{mg}$ AS $115,720 \mathrm{mg}$ AS 155 oder Placebo. Zielvariablen waren das Volumen der Unterschenkel und der Wadenumfang. Bei beiden Parametern verbesserten sich die Werte verglichen mit Placebo signifikant.

Monsieur und Van Snick, 2006

In dieser sechs Wochen andauernden Studie [10] erhielten 39 CVI-Patienten (Schweregrad 2-4) zweimal täglich $180 \mathrm{mg}$ den Weinlaub-Extrakt AS 195. Als Zielparameter diente auch in dieser Studie das Unterschenkelvolumen. Als subjektiver Zielparameter wurde das Schwere- und Schmerzgefühl in den Beinen mittels einer visuellen Analogskala (VAS; $1-10$ ) erhoben. Eine Verbesserung der Symptome konnte schon nach 2 Behandlungswo- chen festgestellt werden. Die Abnahme des Unterschenkelvolumens und die Verbesserung der subjektiven Empfindungen in den Beinen waren am Studienende nach 6 Wochen gegenüber Placebo signifikant.

Diese Studie bestätigte also die Resultate von Kiesewetter et al. [9] und zeigte zudem das schnelle Eintreten der Verbesserung.

\section{Rabe et al., 2011}

Die jüngste klinische Studie über die Wirksamkeit von Weinlaub-Extrakt bei CVI erschien 2011 [11]. Bei dieser 12 Wochen dauernden randomisierten kontrollierten Studie erhielten alle Patienten in der Verum-Gruppe $(\mathrm{n}=126) 720 \mathrm{mg}$ des Weinlaub-Extraktes AS 195. In der Placebo-Gruppe waren 122 Probanden. Die Verminderung des Unterschenkelvolumens in der Verum-Gruppe war verglichen mit der Placebo-Gruppe signifikant grösser. Auch die subjektiven Symptome verbesserten sich in der VerumGruppe klinisch relevant, jedoch nicht signifikant.

\section{Fazit}

Die her vorgestellten Studien dokumentieren die Wirksamkeit von systemisch angewendeten ArzneipflanzenExtrakten zur Behandlung von CVI. Dies gilt in erster Linie für Rosskastanien-Extrakte mit $50 \mathrm{mg}$ Aescin pro Dosis sowie für den standardisierten Extrakt AS 195 aus rotem Weinlaub in einer Dosierung von $360-720$ mg pro Tag.

Damit zeigen sich diese beiden Behandlungsweisen als wirksame und gut verträgliche Therapie der CVI.

\section{Literatur}

1 Beaglehole R: Epidemiology of varicose veins. World J Surg 1986;10:898-902.

2 Bergan JJ, et al: Chronic venous disease. N Engl J Med 2006;355:488-498.

3 Committee on Herbal Medicinal Products (HMPC): EMA/HMPC/228356/2012. 26.01.2017. www.ema.europa.eu/docs/en_GB/document library/Other/2013/08/WC500147372.pdf.

4 Vanscheidt W, et al: Efficacy and safety of a Butcher's broom preparation (Ruscus aculeatus L. extract) compared to placebo in patients suffering from chronic venous insufficiency. Arzneimittelforschung 2002;52:243-250.

5 Uehleke B: Local application of arnica in patients with chronic venous insufficiency due to venous varicosis. 3rd Annual Symposium on Complementary Health Care (Abstract). Forsch Komplementärmed 1996;3:327.

6 Diehm C, et al: Comparison of leg compression stocking and oral horse-chestnut seed extract therapy in patients with chronic venous insufficiency. Lancet 1996;347:292-294.

7 Suter A, et al: An open study to assess the safety and efficacy of Aesculus hippocastanum tablets (Aesculaforce $50 \mathrm{mg}$ ) in the treatment of chronic venous insufficiency. J Herb Pharmacother 2004;4:19-32.

8 Pittler MH, Ernst E: Horse chestnut seed extract for chronic venous insufficiency. Cochrane Database Syst Rev 2012;11:CD003230.

9 Kiesewetter H, et al: Efficacy of orally administered extract of red vine leaf AS 195 (folia vitis viniferae) in chronic venous insufficiency (stages I-II): a randomized, double-blind, placebo-controlled trial. Arzneimittelforschung 2000;50:109-117.
10 Monsieur R, Van Snick G: Action de l'extrait de feuilles de vigne rouge AS 195 dans l'insuffisance veineuse chronique. Praxis (Bern 1994) 2006;95:187-190.

11 Rabe E, et al: Efficacy and tolerability of a redvine-leaf extract in patients suffering from chronic venous insufficiency - results of a double-blind placebo-controlled study. Eur J Vasc Surg 2011;41:540-547. 